\title{
Hybrid filter for object reduction
}

\author{
Mohammed Adam Taheir Mohammed*, Wan Maseri Binti Wan- Mohd, Ruzaini Bin Abdullah Arshah \\ Faculty of Computer System \& Software Engineering, University Malaysia Pahang \\ *Corresponding author E-mail:mohammed_taher30@yahoo.com
}

Copyright $\odot 2014$ Mohammed Adam Taheir Mohammed et al. This is an open access article distributed under the Creative Commons Attribution License, which permits unrestricted use, distribution, and reproduction in any medium, provided the original work is properly cited.

\begin{abstract}
The basic idea to build significant attribute the uncertain objects should remove. Several theories are dealing with uncertainty, soft set theory also handles this uncertainty problem which still an open area to be explored in knowledge management. The propose techniques Known as Filtering data set which used for maintained the inferior object and we need to look at the other side of attribute reduction. The propose technique are reducing the size of object firstly, then the Hybrid reduction are executed for generating the decision extractions. These filters have reduced the size of memory without losing the characteristic of information which absolutely highly efficient. By using Filtering the inferior object of Hybrid techniques are managed. As part of this proposal, an analysis of Hybrid reduction techniques. In the conclusion part Filtering the Hybrid show better result compared to Hybrid reduction.
\end{abstract}

Keywords: Object Reduction; Object Extractions; Parameters Reductions.

\section{Introduction}

How we allocated, no limited resource in storage now a days become an important issue, but they should be balanced when the uncertain object is removed. Discovering false frequent object, especially in attribute reduction take right way for reduction. The question is how much the uncertain object reduces the data set, however, it improves the quality of information as well as storage size are reduced [5]. Handling uncertain data solved by using mathematical principles, and one of them is soft set theory [1]. Soft sets are called "(binary, basic, elementary) neighborhood systems. As for standard soft set, it may be redefined as the classification of objects in two distinct classes, thus confirming that soft set can deal with a Boolean-valued information system". Molotov [1] advantages it is free from the inadequacy of the parameterization tools, unlike in the theories of fuzzy set, probability and interval mathematics. By using this technique the false frequent object is managed and it helps to reduce the numbers of attributes, however, it increases the response time for generating the result. This paper maintains the inferior object of Hybrid reduction techniques [2].

The rest of this paper is organized as follows. Section II presents an analysis of Hybrid reduction. Section III describes the proposed technique and finally, the conclusion part which is described in section IV.

\section{Analysis of hybrid reduction}

Example as mentioned in [5] let "a soft set $(F, E)$ representing studies the communication prowess among selected university student using communication facilities such as "email", $p_{2}$ stands for the parameter "facebook", $p_{3}$ stands for the parameter "blog", $p_{4}$ stands for the parameter "friendsters", $p_{5}$ stands for the parameter "yahoo messenger" and lastly $p_{6}$ stands for the parameter "sms" . Consider the mapping $F: E \rightarrow P(U)$ given by "student communication prowess $(\cdot)$ ", where $(\cdot)$ is to be filled in by one of the parameters $p \in E$ " it represented by Boolean value as shown in table 1 . 
Table 1: Tabular representation of a soft set

\begin{tabular}{llllllll}
\multicolumn{7}{c}{ Table 1: Tabular representation of a soft set } \\
\hline$U / P$ & $p_{1}$ & $p_{2}$ & $p_{3}$ & $p_{4}$ & $p_{5}$ & $p_{6}$ & $f()$. \\
\hline$u_{1}$ & 1 & 0 & 1 & 0 & 0 & 0 & 2 \\
$u_{2}$ & 1 & 1 & 1 & 1 & 1 & 1 & 6 \\
$u_{3}$ & 1 & 1 & 1 & 1 & 1 & 1 & 6 \\
$u_{4}$ & 1 & 1 & 1 & 1 & 1 & 1 & 6 \\
$u_{5}$ & 0 & 1 & 0 & 0 & 1 & 0 & 2 \\
$u_{6}$ & 0 & 0 & 1 & 0 & 1 & 0 & 2 \\
$u_{7}$ & 0 & 0 & 0 & 0 & 1 & 0 & 1 \\
$u_{8}$ & 1 & 1 & 1 & 1 & 1 & 1 & 6 \\
$u_{9}$ & 1 & 1 & 1 & 1 & 1 & 1 & 6 \\
$u_{10}$ & 1 & 1 & 1 & 1 & 1 & 1 & 6 \\
$u_{11}$ & 1 & 0 & 1 & 0 & 1 & 0 & 3 \\
$u_{12}$ & 0 & 1 & 0 & 1 & 0 & 0 & 2 \\
$u_{13}$ & 1 & 1 & 1 & 1 & 1 & 1 & 6 \\
$u_{14}$ & 1 & 1 & 0 & 0 & 0 & 0 & 2 \\
$u_{15}$ & 1 & 1 & 1 & 1 & 1 & 1 & 6 \\
$u_{16}$ & 1 & 1 & 1 & 1 & 1 & 1 & 6 \\
$u_{17}$ & 1 & 1 & 1 & 1 & 1 & 1 & 6 \\
$u_{18}$ & 0 & 0 & 1 & 1 & 1 & 0 & 3 \\
\hline
\end{tabular}

\subsection{The procedure of hybrid reduction in soft set decision making [2]}

1) Input soft set (F, E) over universe $U$.

2) Determine which attributes have true value for every object.

3) Calculate the support co-occurrences.

4) Find optimal, sub optimal result until last result.

5) Determine the order of supports by arranging in decreasing order.

6) Rank the result until the inferior object based on support.

7) Find the U/E clusters partions.

8) Determine the optimal decision partions and $\operatorname{supp}_{A}(u)=\operatorname{supp}(v)$ A for every $u, v$ in $U$.

9) For any group of attributes satisfies procedure 6 determine the reduction.

10) Select any row fulfills the definition of ultimate support set.

11)Delete the object partition which in ultimate support.

12)For any ultimate minimum support deletes the partions of inferior object.

13) If there is any ultimate minimum support set, mark the object the inferior object.

14) Remove every row or columns which have a no object (zero significant).

The parameters co-occurrence set is the representation of the true value which as Coo $\mathrm{u} 1=\{\mathrm{e} 1, \mathrm{e} 3\}$ then Coo u2 until last object and the result of reductions based on table 1 as shown in table2.

Table 2: shows figure 1 soft set

\begin{tabular}{llllllll}
\hline$U / P$ & $p_{1}$ & $p_{2}$ & $p_{3}$ & $p_{4}$ & $p_{5}$ & $p_{6}$ & $f()$. \\
\hline$u_{1}$ & 1 & 0 & 1 & 0 & 0 & 0 & 2 \\
$u_{5}$ & 0 & 1 & 0 & 0 & 1 & 0 & 2 \\
$u_{6}$ & 0 & 0 & 1 & 0 & 1 & 0 & 2 \\
$u_{7}$ & 0 & 0 & 0 & 0 & 1 & 0 & 1 \\
$u_{11}$ & 1 & 0 & 1 & 0 & 1 & 0 & 3 \\
$u_{12}$ & 0 & 1 & 0 & 1 & 0 & 0 & 2 \\
$u_{14}$ & 1 & 1 & 0 & 0 & 0 & 0 & 2 \\
$u_{18}$ & 0 & 0 & 1 & 1 & 1 & 0 & 3 \\
\hline
\end{tabular}




\section{Propose techniques}

In this section, filtering the Hybrid reduction main idea is to further reduce the size of database by removing the false frequent object which managed the inferior object to achieve the quality of information without losing the characteristic of the original data set as shown in fig.1.

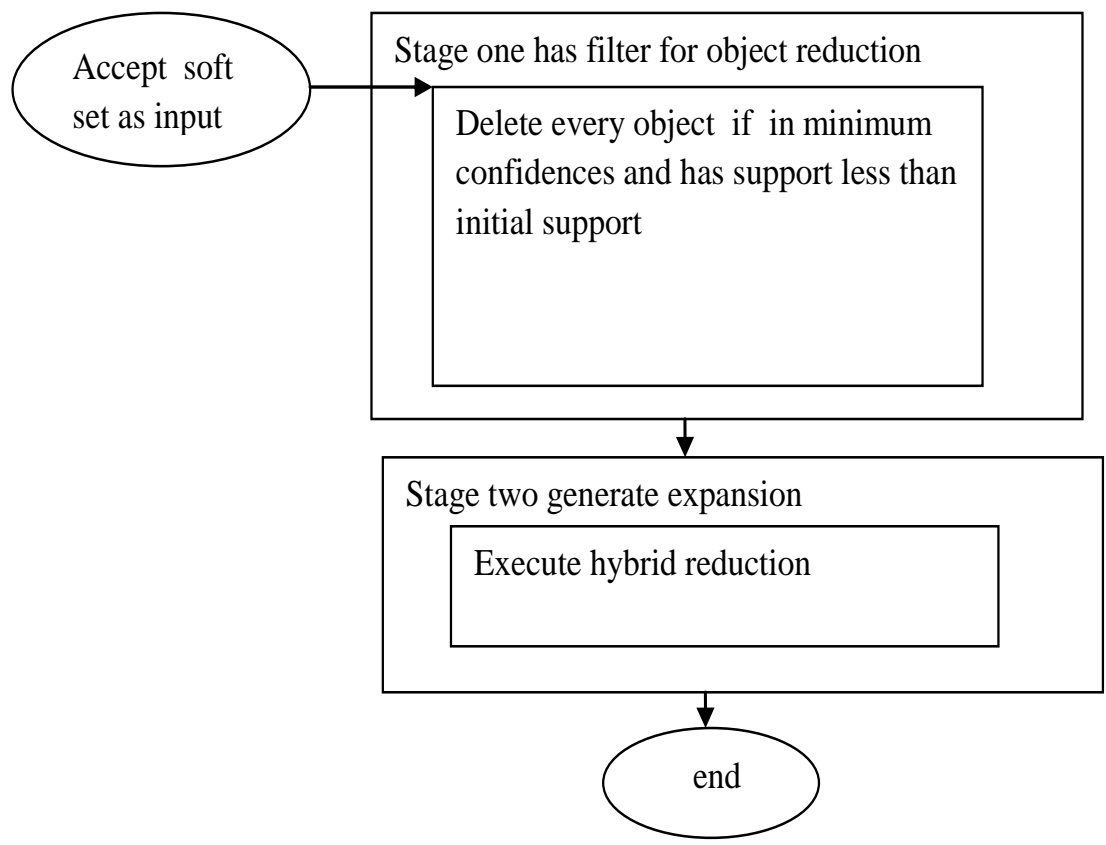

Fig. 1: the procedure of Filtering the Hybrid reduction technique.

\subsection{The proposed procedure as follows}

1) Accept the input from the original data set.

2) Calculate every object supports.

3) Determine the minimum support partions.

4) Calculate the object confidences of minimum support partions.

5) Delete the object from the data set if in minimum confidences and their support are less than predefined support.

6) Execute Hybrid reduction

7) This procedure Known as Filtering the Hybrid Reduction

\subsection{Analysis of filtering the Hybrid reduction}

The association rule which safeties the confidence mentioned in [4]. After filtering table 1 by this technique every object not in maximum optimal partions is selected and the result as shown in table 2.

Based on table 2 [3] we calculate the confidence of support [4] there for the object $\mathrm{u} 7$ in minimum support partion and the confidence of $\mathrm{u} 7=0 \%$ in table 1 the object $\mathrm{u} 7$ is deleted in part one filtering because in minimum support portion the result as shown in table 3.

Then the result is generated based on Hybrid reduction as shown in table 4 .

\section{Conclusion}

Organizing everything is time consuming, but best if their result is enhanced. Removing false frequent object affect the attribute reduction directly it need to manage carefully. By using this technique every object in minimum confidences and their support less than initial support then the object is not forwarded to Hybrid reduction techniques which enhanced the data set size reduction to this the false frequent object are managed, this technique increased the response time of Hybrid reduction as well as the inferior object are managed because in reality the uncertain data become a big deal. This technique reduces the object size drastically, but still being able to maintain consistency in decision making. 
Table 3: shows the object filtered in stage one

\begin{tabular}{llllllll}
\hline$U / P$ & $p_{1}$ & $p_{2}$ & $p_{3}$ & $p_{4}$ & $p_{5}$ & $p_{6}$ & $f()$. \\
\hline$u_{1}$ & 1 & 0 & 1 & 0 & 0 & 0 & 2 \\
$u_{2}$ & 1 & 1 & 1 & 1 & 1 & 1 & 6 \\
$u_{3}$ & 1 & 1 & 1 & 1 & 1 & 1 & 6 \\
$u_{4}$ & 1 & 1 & 1 & 1 & 1 & 1 & 6 \\
$u_{5}$ & 0 & 1 & 0 & 0 & 1 & 0 & 2 \\
$u_{6}$ & 0 & 0 & 1 & 0 & 1 & 0 & 2 \\
$u_{8}$ & 1 & 1 & 1 & 1 & 1 & 1 & 6 \\
$u_{9}$ & 1 & 1 & 1 & 1 & 1 & 1 & 6 \\
$u_{10}$ & 1 & 1 & 1 & 1 & 1 & 1 & 6 \\
$u_{11}$ & 1 & 0 & 1 & 0 & 1 & 0 & 3 \\
$u_{12}$ & 0 & 1 & 0 & 1 & 0 & 0 & 2 \\
$u_{13}$ & 1 & 1 & 1 & 1 & 1 & 1 & 6 \\
$u_{14}$ & 1 & 1 & 0 & 0 & 0 & 0 & 2 \\
$u_{15}$ & 1 & 1 & 1 & 1 & 1 & 1 & 6 \\
$u_{16}$ & 1 & 1 & 1 & 1 & 1 & 1 & 6 \\
$u_{17}$ & 1 & 1 & 1 & 1 & 1 & 1 & 6 \\
$u_{18}$ & 0 & 0 & 1 & 1 & 1 & 0 & 3 \\
\hline
\end{tabular}

The result of this proposes reduction as shown in table 4.

Table 4

\begin{tabular}{llllll}
\hline$U / P$ & $p_{1}$ & $p_{4}$ & $p_{5}$ & $p_{6}$ & $f()$. \\
\hline$u_{1}$ & 1 & 0 & 0 & 0 & 2 \\
$u_{5}$ & 0 & 0 & 1 & 0 & 2 \\
$u_{6}$ & 0 & 0 & 1 & 0 & 2 \\
$u_{11}$ & 1 & 0 & 1 & 0 & 3 \\
$u_{12}$ & 0 & 1 & 0 & 0 & 2 \\
$u_{14}$ & 1 & 0 & 0 & 0 & 2 \\
$u_{18}$ & 0 & 1 & 1 & 0 & 3 \\
\hline
\end{tabular}

\section{References}

[1] D.Molotov, "Soft set theory-first results", Computers and Mathematics with Applications, vol.37, pp.19-31, 1999.

[2] A.M.M.Rose, M.I.Awang, H.Hassan, A.H.Zakaria, T.Herawan, and M.M.Deris, "Hybrid Reduction in Soft Set Decision Making", Springer, pp. 108-115, 2011.

[3] M.A.T.Mohammed,W.M.B.W.Mohd, R.B.A.Arshah, L.Yao, " Predefined Object Reduction”, International Journal of Advanced Research in Computer Engineering \& Technology (IJARCET), Vol. 2, Issue 12 December 2013, pp. 2278 - 1323.

[4] V.Rajpoot, Prof. S.k.Shrivastava, Prof. A.Mathur, “An Efficient Constraint Based Soft Set Approach for Association Rule Mining ", IJERA, Vol. 2 issue 4 July-August 2012, pp.2210-2215.

[5] M.A.T.Mohammed,W.M.B.W.Mohd, R.B.A.Arshah, L.Yao, "parameter reduction comparision”, Asian Academic Research Associates, vol.1 issue 19 January 2014. 